\title{
MRI IMAGES THRESHOLDING FOR ALZHEIMER DETECTION
}

\author{
Ali El-Zaart and Ali A.Ghosn \\ Department of Mathematics and Computer Science, \\ Beirut Arab University, Beirut, Lebanon \\ elzaartebau.edu.lb, sawaghosn@hotmail.com
}

\begin{abstract}
More than 55 illnesses are associated with the development of dementia and Alzheimer's disease $(A D)$ is the most prevalent form. Vascular dementia (VD) is the second most common form of dementia. Current diagnosis of Alzheimer disease (Alzheimer's disease) is made by clinical, neuropsychological, and neuroimaging assessments. Magnetic resonance imaging (MRI) can be considered the preferred neuroimaging examination for Alzheimer disease because it allows for accurate measurement of brain structures, especially the size of the hippocampus and related regions. Image processing techniques has been used for processing the (MRI) image. Image thresholding is an important concept, both in the area of objects segmentation and recognition. It has been widely used due to the simplicity of implementation and speed of time execution. Many thresholding techniques have been proposed in the literature. The aim of this paper is to provide formula and their implementation to threshold images using Between-Class Variance with a Mixture of Gamma Distributions. The algorithms will be described by given their steps, and applications. Experimental results are presented to show good results on segmentation of (MRI) image.
\end{abstract}

\section{KEYWORDS}

MRI Images, Image Thresholding, Between class variance, Gamma distribution.

\section{INTRODUCTION}

Alzheimer's disease (AD) is a progressive, degenerative disorder that attacks the brain's nerve cells, or neurons, resulting in loss of memory, thinking and language skills, and behavioral changes. As life span is increasing, an early detection of $\mathrm{AD}$ emerges as possible approach to delay its consequences on patients and to increase the chance of getting potential benefits from approved medications and maintain certain good level of life. In 2006, the worldwide prevalence of $\mathrm{AD}$ was 26.6 million cases, and it was predicted that it will grow to 106.8 million in 2050 . Among these, there were 12.6 million cases in Asia (48\% of worldwide prevalence), and it was predicted that it will grow to 62.85 (59\% of worldwide prevalence) in $2050 .{ }^{3}$

Routine structural neuroimaging evaluation is based on nonspecific features such as atrophy, which is a late feature in the progression of the disease. Therefore, developing new approaches 
for early and specific recognition of Alzheimer disease at the prodromal stages is of crucial importance.

Segmentation is an important issue in MRI image processing for diagnosis of (AD). One of the simplest methods for image segmentation is thresholding [6]. It is based on selection of a value that separates between two classes of gray-level values; this selected value is called threshold value; which classify the gray-level value into two ranges to separate the object from the background. The difficulty placed in determining the right threshold value or the optimal one. Threshold techniques can be classified mainly into six groups according to the information they are exploiting $[8,9,10,11,12,13,14]$. They are:

1) Histogram-based Methods. 2) Clustering-based Methods. 3) Object attribute-based Methods. 4) Spatial Methods. 5) Local Methods 6) Entropy-based Methods.

However, this work's goal is improving Otsu's method [6,7], so it can estimate the optimal threshold values of images based on Gamma distribution, by using iteratively algorithm. Otsu's method is weak when it comes to dealing with low contrast images or where the object is small as in [3]. In general, Otsu's method produces a threshold value that maximizes between-class variances. This work focuses on improving Otsu's thresholding method to generate threshold value automatically for a grayscale images based on Gamma distribution. Symmetric and asymmetric histogram distribution of the intensity values can be represented by using Gamma distribution [5]. Gamma distribution is extensively more than Gaussian distribution, which only represents symmetric histogram distribution of the intensity values.

For each technique let $f(i, j)$ is the original image, $T$ is the threshold value, and $g(i, j)$ is the output image:

$$
g(i, j)= \begin{cases}1, & \text { for } f(i, j) \geq T \\ 0, & \text { otherwise }\end{cases}
$$

This paper will explore the related work in the field of image thresholding segmentation while focusing on Otsu's method. Section 2, demonstrates Gamma distribution. Our proposal method explained in section IV. And in section V, experimental results show the efficiency of our method in both bimodal and multilevel thresholding. Finally, in section VI will give the conclusion and future works.

\section{OTSU METHOD}

Otsu's method [1] is one of the most popular methods for its simplicity and efficiency [4]. It is based in thresholding technique. It depends on selecting the optimal threshold value that maximizes the between-class variance of resulting object and background classes. The search for the optimal threshold done sequentially until finding a value that makes variance between two classes or more maximum. In this section, will demonstrate Otsu's method and its development which made it fast in computational. If the image in the two dimensions is represented by the function $\mathrm{f}(\mathrm{x}, \mathrm{y})$ and the values of it in gray-level have the range between [0...L], where $\mathrm{L}=255$. And let $\mathrm{N}$ represent the whole number of pixels in the image. The number of pixels with graylevel $\mathrm{i}$ ish(i), $\mathrm{i}=[0,1, \ldots, \mathrm{L}]$ that represent the histogram of the image. For the simplest, normalize 
of the histogram was computed which represents the probability ofoccurrence of gray-level $i$ as the follows: $\mathrm{p}(\mathrm{i})=\frac{\mathrm{h}(\mathrm{i})}{\mathrm{N}}, \mathrm{p}(i) \geq 0$

The total average or mean value of the image computed as:

$$
\mu_{T}=\sum_{i=0}^{\mathrm{L}-1} \operatorname{ip}(i) \sum_{i=0}^{\mathrm{L}-1} \mathrm{p}(i)=1
$$

For the single thresholding, image pixels will be divided into two classesC $1\{0,1, \ldots, \mathrm{t}\}$, and $\mathrm{C} 2\{\mathrm{t}, \ldots, \ldots, \mathrm{L}-1\}$, where tis the threshold value. Usually, the resulted image from this method corresponds to separate the object (C2thatrepresents the class of bright pixels) from the background ( $\mathrm{C} 1$ that represents the classof dark pixels). The probability of these two classes is:

$$
\omega_{1}(t)=\sum_{i=0}^{t-1} p(i) \operatorname{and} \omega_{2}(t)=\sum_{i=t}^{L} p(i)
$$

And the mean for them are:

$$
\begin{aligned}
& \mu_{1}(t)=\sum_{i=0}^{t} i h(i) / \omega_{1}(t) \\
& \mu_{2}(t)=\sum_{i=t}^{L-1} i h(i) / \omega_{2}(t)
\end{aligned}
$$

Then by using discriminate analysis [1], Otsu proves that optimal threshold ( $\left.\mathrm{t}^{*}\right) \mathrm{can}$ be obtained by maximizing the between-class variance as:

$$
\mathrm{t}^{*}=\operatorname{Arg} \max _{0 \leq \mathrm{t} \leq 255}\left\{\sigma_{\mathrm{B}}^{2}(\mathrm{t})\right\}
$$

Where the between-class variance $\left(\sigma_{\mathrm{B}}^{2}(\mathrm{t})\right)$ is defined as:

$$
\begin{gathered}
\eta(t)=\sigma_{B}^{2}(t) \\
\sigma_{B}^{2}(t)=\omega_{1}(t)\left(\mu_{1}(t)-\mu_{T}\right)^{2}+\omega_{2}(t)\left(\mu_{2}(t)-\mu_{T}\right)^{2}
\end{gathered}
$$

This method also has been extended to multilevel thresholding of image by Otsu [1]. If we have M classes where:

$\mathrm{C}_{1}=\{0, . ., \mathrm{t} 1\}, \mathrm{C}_{2}=\left\{\mathrm{t}_{1}, . ., \mathrm{t}_{2}\right\}$ and $\mathrm{CM}=\left\{\mathrm{t}_{\mathrm{M}-1}+1, . ., 255\right\}$

Then the threshold values will be:

$$
\left\{t_{1}^{*}, t_{2}^{*}, \ldots \ldots t_{\mathrm{M}-1}^{*}\right\}=\operatorname{Arg} \max _{0 \leq \mathrm{t} \leq 255}\left\{\sum_{k=1}^{M} \omega_{k}\left(\mu_{k}-\mu_{T}\right)^{2}\right\}
$$

Many papers have been published which study the improvement of this method [7, 21]. In [2], the author improves Otsu's method to make it more efficient in computational side. He used the derivation computation to find the optimal threshold value in iteratively manner rather than 
searching in sequential that decrease the loop, and then the time of CPU needed for the computational process. He concluded to the following formula for two classes:

$$
t=\frac{\mu_{1}+\mu_{2}}{2}
$$

Where $\mu 1$ is the mean of the first class and $\mu 2$ is the mean for second class. This formula has convergence property [56] which means that in each iteration the threshold value converges to be optimal.

In [7], the authors also developed algorithm to compute multilevel threshold values faster, using recursive algorithm with look-up table that shortens the huge number of mathematical operations needed in this situation.

However, this method is only applied when the histogram is symmetric but for asymmetric histogram it is better to use Gamma distribution as described in the next section.

\section{GAMMA DISTRIBUTION}

Histogram represents statistical information for image pixels. It describes pixels intensity distribution in an image by graphing the number of pixels intensity at each gray level or color intensity level. Essentially, we can say that there are two types of distributions of gray level (mode): symmetric and non-symmetric. For the symmetric mode Gaussian distribution work well to estimate the threshold value. On the other hand, Gamma distribution is more general to represent the both symmetric and non-symmetric modes. The Gamma function defines as [5]:

$f(x, \mu, N)=\frac{2 q}{\mu} \frac{N^{N}}{\Gamma(N)}\left[\frac{q x}{\mu}\right]^{2 N-1} e^{-N\left(\frac{q x}{\mu}\right)^{2}}$

Where $q=\Gamma(N+0.5) / \sqrt{N} \Gamma(N), x$ is the intensity of the pixel, $\mu$ is the mean value of the distribution and $\mathrm{N}$ is the shape of distribution. In our method, Gamma distribution used to estimate the mean values of the image modes and then find the optimal threshold value. Figure 1, shows the Gamma distribution for one mode with different shape parameter $\mathrm{N}$ and same value of mean $\mu$.

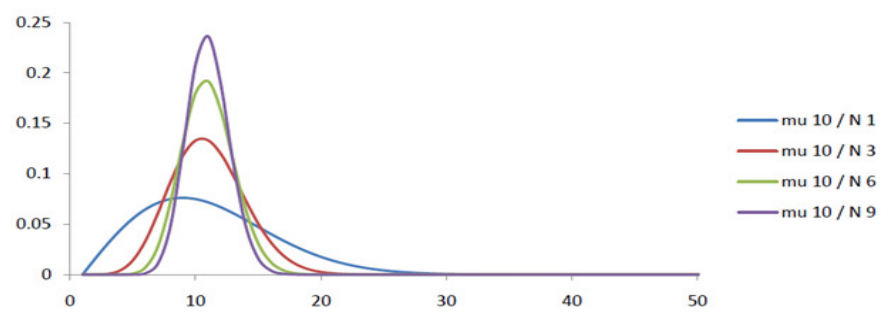

Figure 1: Gamma Distribution with the same mean and ditterent snape parameter N.

\section{VALLEY-EMPHASIS METHOD USING A MiXTURE OF GAMMA DISTRIBUTIONS FOR SMALL OBJECT}

This Section will demonstrate our proposal method. It solves the problem of image segmentation in case if it contains small object. As mentioned before in section 3, this method also based onOtsu's method using Gamma distribution. We improved our method and gave it the ability to deal with small objects. As well as it can deal with asymmetric mode in the histogram to get more 
satisfying segmentationresults. We give comprehensive explain on the bimodal and multimodal thresholding algorithms.

\subsection{Bimodal Thresholding}

Hui-Fuang suggested adding a weight with Otsu's method for detecting small object [3]. The author observes when select the threshold value to be on the valley of the two peaks (from the histogram), the probability of occurrence at the threshold value (pt) has to be small. Based on this observation he proposed to add the weight to Otsu's method which will improve it for selecting threshold values. However, this method called "valley-emphasis". Objective to select a threshold value that has small probability of occurrence (valley in the gray-level histogram), and it also maximizes the between class variance, as in the Otsu method [3]. By applying the weight (1-pt) to Otsu's method is the key of the valley-emphasis method. The smaller the $(\mathrm{p}(\mathrm{t}))$ value, the larger the weight (1-pt) will be. This weight ensures that the result threshold value will always be located in at the valley or bottom rim of the gray-level distribution [3].As Otsu's method, the valley-emphasis method also attempts to maximize the between-class variance of the histogram. Based on Hui-Fuang's work we proposed our method for bimodal thresholding as the following:

$\eta(t)=\sigma_{B}^{2}(t)=\left[\omega_{1}(t)\left(\mu_{1}(t)-\mu_{T}\right)^{2}+\omega_{2}(t)\left(\mu_{2}(t)-\mu_{T}\right)^{2}\right](1-p(t))$

Where the probability of occurrence of gray-level $t$ is defined as:

$p(t)=\frac{\mathrm{h}(\mathrm{t})}{\mathrm{n}} ; \quad \mathrm{P}^{\prime}(t)=\frac{\mathrm{h}(\mathrm{t})-\mathrm{h}(\mathrm{t}-1)}{\mathrm{n}}$

$\mathrm{n}$ is the total number of pixels in a given image. $\mathrm{P}^{\prime}(\mathrm{t})$ is the first derivative of the probability.

We consider $h(i), i=0 \ldots 255$ be the bimodal histogram of the original image. We assume that the histogram of an image can be seen as a combination of Gamma distributions. The mean values of each mode can be estimated using Gamma distribution [5]. We used Gamma distribution because it has the ability to represent both symmetric and non-symmetric mode rather than the limited Gaussian distribution that describes only the symmetric mode better. However, the mean values will be as the following:

$$
\begin{aligned}
& \mu_{1}(t)=\sqrt{\frac{\sum_{i=0}^{t-1} h(i) i^{2} q^{2}}{\sum_{i=0}^{t-1} h(i)}}=\frac{\sqrt{\sum_{i=0}^{t-1} h(i) i^{2} q^{2}}}{\sqrt{\omega_{1}(t)}}=\frac{\mu_{1 a}(t)}{\mu_{0 a}(t)}(5) \\
& \mu_{2}(t)=\sqrt{\frac{\sum_{i=t}^{L} h(i) i^{2} q^{2}}{\sum_{i=t}^{L} h(i)}}=\frac{\sqrt{\sum_{i=t}^{L} h(i) i^{2} q^{2}}}{\sqrt{\omega_{2}(t)}}=\frac{\mu_{1 b}(t)}{\mu_{0 b}(t)}(6)
\end{aligned}
$$

Where $\mu \mathrm{T}$ the total mean of image, $\mu 1$ the mean value of the first class and $\mu 2$ the mean of the second class.h(i), $\mathrm{I}=0 \ldots 255$ is the histogram of image. Here, we aim to divide the histogram in two classes $\mathrm{C} 1\{0,1, \ldots, \mathrm{t}\}$, and $\mathrm{C} 2\{\mathrm{t}+1, \ldots, 255\}$. Where $\mathrm{t}$ is the threshold value. Consequently we can define the thresholded image as:

$g(x, y)= \begin{cases}0 & f(x, y) \leq t \\ 255 & f(x, y)>t\end{cases}$ 
Where $f(x, y)$, is the original image, and $g(x, y)$ is the segmented image result.

Therefore, we calculate the first derivative of Eq. (4) then we set it to zero [2] to get the optimal threshold value as:

$\left[\omega_{1}(t)\left(\mu_{1}(t)-\mu_{T}\right)^{2}+\omega_{2}(t)\left(\mu_{2}(t)-\mu_{T}\right)^{2}\right]^{\prime}(1-p(t))+\left[\omega_{1}(t)\left(\mu_{1}(t)-\mu_{T}\right)^{2}+\right.$ $\left.\omega_{2}(t)\left(\mu_{2}(t)-\mu_{T}\right)^{2}\right](p(t)-1)^{\prime}=0$ The first derivative of Eq. (2) is:

$\left[\boldsymbol{\omega}_{\mathbf{1}}(\boldsymbol{t})\left(\boldsymbol{\mu}_{\mathbf{1}}(\boldsymbol{t})-\boldsymbol{\mu}_{\boldsymbol{T}}\right)^{\mathbf{2}}+\boldsymbol{\omega}_{2}(\boldsymbol{t})\left(\boldsymbol{\mu}_{\mathbf{2}}(\boldsymbol{t})-\boldsymbol{\mu}_{\boldsymbol{T}}\right)^{2}\right]^{\prime}=\left[\omega_{1}^{\prime}(t)\left(\mu_{1}(t)-\mu_{T}\right)^{2}+2 \omega_{1}(t)\left(\mu_{1}(t)-\right.\right.$ $\left.\left.\mu_{T}\right) \mu_{1}^{\prime}+\omega_{2}^{\prime}(t)\left(\mu_{2}(t)-\mu_{T}\right)^{2}+2 \omega_{2}(t)\left(\mu_{2}(t)-\mu_{T}\right) \mu_{2}^{\prime}(t)\right](8)$

$$
\begin{gathered}
\omega_{1}^{\prime}(t)=h(t) ; \omega_{2}^{\prime}(t)=-h(t) \\
\mu_{1}^{\prime}(t)=\frac{h(t)}{2 \mu_{0 a}(t)}\left[\frac{t^{2} q^{2}}{\mu_{1 a}(t)}-\frac{\mu_{1}(t)}{\mu_{0 a}(t)}\right] \\
\mu_{2}^{\prime}(t)=\frac{-h(t)}{2 \mu_{0 b}(t)}\left[\frac{t^{2} q^{2}}{\mu_{1 b}(t)}-\frac{\mu_{2}(t)}{\mu_{0 b}(t)}\right] \\
\mu_{1 a}^{\prime}(t)=\frac{h(t) t^{2} q^{2}}{2 \mu_{1 a}(t)} ; \quad \mu_{0 a}^{\prime}(t)=\frac{h(t)}{2 \mu_{0 a}(t)} \\
\mu_{1 b}^{\prime}(t)=-\frac{h(t) t^{2} q^{2}}{2 \mu_{1 b}(t)} ; \quad \mu_{0 b}^{\prime}(t)=-\frac{h(t)}{2 \mu_{0 b}(t)}
\end{gathered}
$$

Equation (8) will lead to

Where

$$
A(t)+B(t) t^{2}=0
$$

$$
\begin{gathered}
\mathrm{A}(\mathrm{t})=\mathrm{h}(\mathrm{t})(\mathrm{n}-\mathrm{h}(\mathrm{t})) \mu_{\mathrm{T}}\left(\mu_{2}(\mathrm{t})-\mu_{1}(\mathrm{t})\right)-[\mathrm{h}(\mathrm{t})-\mathrm{h}(\mathrm{t}-1)]\left[\omega_{1}(\mathrm{t})\left(\mu_{1}(\mathrm{t})-\mu_{\mathrm{T}}\right)^{2}+\right. \\
\left.\quad \omega_{2}(\mathrm{t})\left(\mu_{2}(\mathrm{t})-\mu_{\mathrm{T}}\right)^{2}\right](13) \\
B(t)=h(t) q^{2}(n-h(t))\left[\frac{1}{\mu_{2}(t)}-\frac{1}{\mu_{1}(t)}\right] \\
\quad \because A(t)+B(t) t^{2}=0 \rightarrow \\
\therefore t=+\sqrt{\frac{-A(t)}{B(t)}} ; \\
\mathrm{B}(\mathrm{t}) \neq 0 \text { and } \frac{-\mathrm{A}(\mathrm{t})}{\mathrm{B}(\mathrm{t})} \geq 0
\end{gathered}
$$

From the Eq. (15), we will get the optimal threshold value (t).

This formula allows us to use an iterative thresholding algorithm that makes the new threshold value converge to the optimal threshold value and decrease the processing time of calculation. The bimodal thresholding algorithm of thismethod is described in as: 
1. Inputs: image $f(x, y)$

2. Compute histogram of input image $h(i), i=0.255$.

3. Compute initial threshold value as a total mean of the image $\left(q^{2}=0.98\right)$ :

4. Compute $\mu_{1}(\mathrm{t})$ and $\mu_{2}(\mathrm{t})$ in Eq. $(5,6)$.

$$
t_{0}=\sqrt{\frac{\sum_{i=0}^{255} h(i) i^{2} q^{2}}{\sum_{i=0}^{255} h(i)}}
$$

5. Compute tnew using equation 15 .

6.

$$
\begin{aligned}
& \text { If } \mid t 0 \text { - tnew } \mid<=0.1 \text { Then } \\
& \text { Find the optimal threshold value }{ }^{*} \\
& \text { Else }
\end{aligned}
$$

\section{Assign t0 $\leftarrow$ tnew}

Go to step 4 .

7. Perform the thresholding process as in Eq. (7) according to the optimal threshold value $t^{*}$ taken from step 6.

\subsection{Multimodal Thresholding}

In multilevel thresholding, the resulted image will be segmented to $\mathrm{M}$ mode according to a set of threshold values $\mathrm{T}$, where $\mathrm{T}=\{\mathrm{t} 1, \mathrm{t} 2, \mathrm{t} 3, \ldots . ., \mathrm{tM}-1\}$, where:

$\mathrm{t} 0=0<\mathrm{t} 1<\mathrm{t} 2<\mathrm{t} 3<\ldots \ldots<\mathrm{tM}-1<\mathrm{tM}=255$. The means of each mode (classes) or the $\mathrm{kth}$ mode defined as in Eq. (16).

$$
\begin{aligned}
& \mu_{k}(t)=\sqrt{\frac{\sum_{i=t_{k-1}}^{t_{k}} h(i) i^{2} q^{2}}{\sum_{i=t_{k-1}}^{t_{k}} h(i)}} \\
& k=1,2 \ldots . ., M
\end{aligned}
$$

The optimal threshold value $T^{*}=\left\{t_{1}^{*}, t_{2}^{*}, t_{3}^{*}, \ldots, t_{M-1}^{*},\right\}$ for each class will be estimated by using the bimodalthresholding mention in section 4.A. The multilevel thresholding algorithm is described as:

1. Inputs: image $f(x, y)$ and number of modes $M$

2. Compute histogram of input image $h(i), i=0.255$.

3. Compute initial threshold values by using $\mathrm{k}-$ mean algorithm $\quad \mathrm{T} 0=\left\{\mathrm{t}_{0}^{1}, \mathrm{t}_{0}^{2}, \mathrm{t}_{0}^{3}, \ldots, \mathrm{t}_{0}^{\mathrm{M}-1}\right\}$.

4. Compute the new threshold values using bimodalmethod

where $\mathrm{k}=1,2 \ldots \mathrm{M}-1$

$$
{ }^{\mathrm{k}}{ }_{\text {new }}^{\mathrm{k}}=\operatorname{bimodal}\left(\mathrm{t} \mathrm{k}_{0}, \quad \mathrm{t} \underset{0}{\mathrm{k}+1}, \mathrm{~h}(\mathrm{t})\right)
$$

5. If $\left|t_{\text {new }}^{1}-t_{0}^{1}\right|>0.1$ OR .. OR $\left|t_{\text {new }}^{\mathrm{M}-1}-t_{0}^{\mathrm{M}-1}\right|>0.1$ Then

Assign T0 Tnew

Go to step 4 .

Else

Find the optimal threshold values $\mathrm{T}^{*}$ 


\section{EXPERIMENTAL RESULTS}

This section provides experimental results for applying our valley-emphasis method on MRI images. Figure 2 shows an original MRI images and the segmentation result.
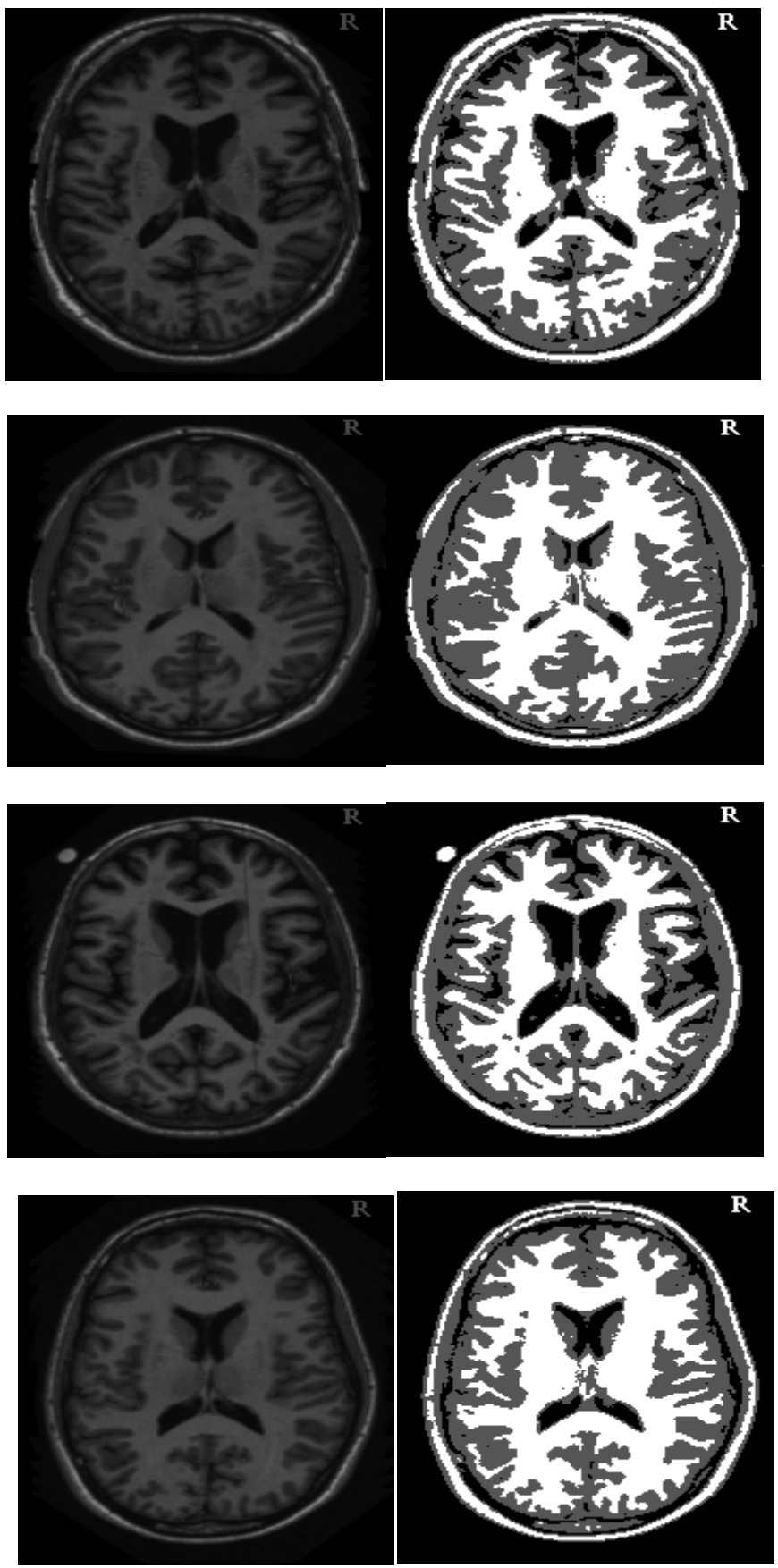


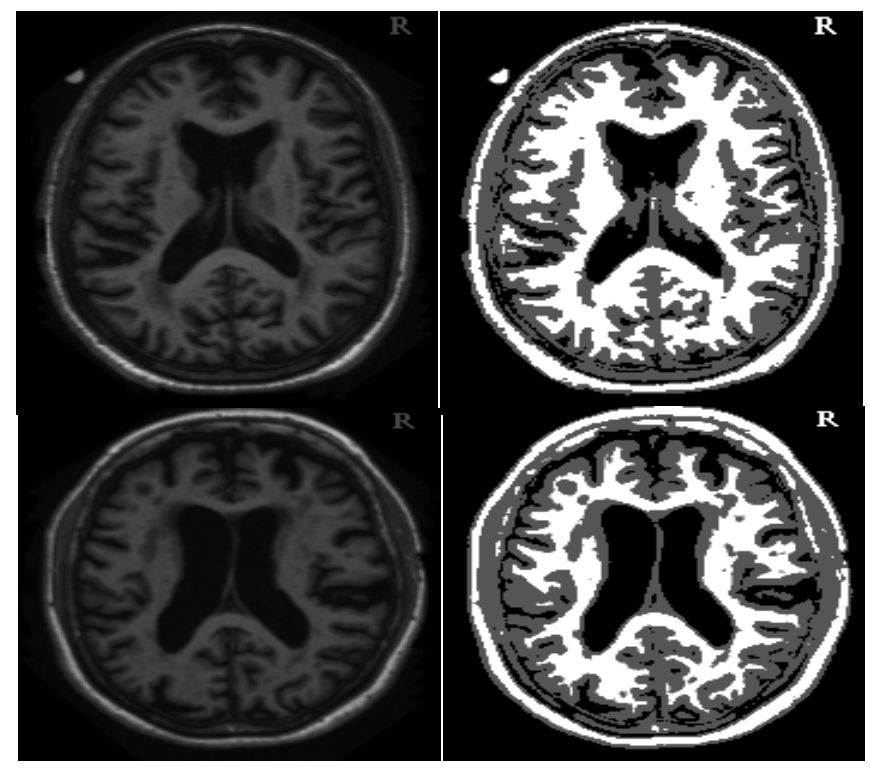

Figure 2. Original MRI images and segmented Results.

\section{CONCLUSiOnS}

We have proposed a new method for image thresholding (bimodal and multimodal thresholding) based on between-class variance and using Gamma distribution. Our method solves the problem of non-symmetric histogram of images by using Gamma rather than Gaussian distribution. We have also applied it iteratively to make the threshold value converges to be optimal value, and to reduce the computations. In addition, we provided experimental results that show our method efficiency in comparison with the original Gaussian Otsu method. In addition we extend our proposed method to solve the problem for detecting the small object in the images. As well as shown in the experimental results we have satisfied segmentation results. Finally we applied our algorithm in many other images as medical images.

\section{REFERENCES}

[1] N.Otsu, "A threshold selection method from gray-level histogram," IEEE Transactions on Systems, MAN, and Cybernetics, Vol. SMC-9, No. 1, 1979, pp. 62-66.

[2] K.Chin Lin, "Fast thresholding computation by searching for zero derivatives of image BetweenClass Variance," the 27th Annual Conference of the IEEE Industrial Electronics Society, 2001, pp. 393-397.

[3] H.Fuang Ng, "Automatic Thresholding for Defect Detection," Pattern Recognition Letters, Vol. 27, 2006, pp.1644-1649.

[4] Z.Hou, Q. Hu and W.L. Nowinski, "On minimum variance thresholding," Pattern Recognition Letters, Vol. 27, 2006, pp. 1732-1743.

[5] A.El Zaart, D. Ziou, S. Wang and Q. Jiang, " Segmentation of SAR images," Pattern Recognition Journal, Vol. 35, No. 3, 2002, pp. 713-724.

[6] J.C. Russ, "The image processing handbook," CRC Press, Boca Raton, NW, USA, 5th edition, 2007.

[7] P.Liao, T. Chen, and P. Chung, "A Fast Algorithm for Multilevel Thresholding," Journal of Information Science and Engineering, Vol. 17, 2001, pp. 713-727. 
[8] T.Logeswari ,M. Karnan (2010). An improved implementation of brain tumor detection using segmentation based on soft computing. J. Cancer Res. Exp. Oncol., 2(1): 006-014.

[9] K.Mirzaei, H. Motameni, R. Enayatifar (2011). New method for edge detection and de noising via fuzzy cellular automata. Int. J. Phy. Sci. 6(13): 3175-3180.

[10] P.Sathya, R. Kayalvizhi(2010). PSO-Based TsallisThresholdingSelection Procedure for Image Segmentation. Int. J. Comput. Appl.5(4): 39-46.

[11] O.Sojodishijani, V .Rostam, A. Ramli (2010). A video-rate color image segmentation using adaptive and statistical membership function. Sci. Res. Essays, 5(24): 3914-3925.

[12] L.Yu (2008). Vehicle extractions using histograms and genetic algorithm based fuzzy image segmentation from high resolution UAV aerial imagery. The International Archives of the Photogrammetric, Remote Sens. Spat.

[13] E.Arias-Castro, DL. Donoho (2009). Does median filtering truly preserve edges better than linear filtering? Annals of Statistics. 37(3): 1172-2009.

[14] Y.Fernandez, A. Sarinana, R. Swenson (2009). development of a prototype for classification of potato mini-tubers based on artificial vision. 6th International Conference on Electrical Engineering, Computing Science and Automatic Control.

\section{AUTHOR}

Ali El-Zaart was a senior software developer at Department of Research and Development, Semiconductor Insight, Ottawa, Canada during 2000-2001. From 2001 to 2004, he was an assistant professor at the Department of Biomedical Technology, College of Applied Medical Sciences, King Saud University. From

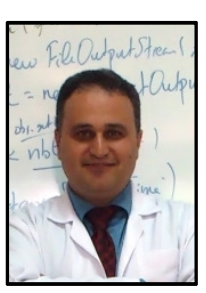
2004-2010 he was an assistant professor at the Department of Computer Science, College of computer and information Sciences, King Saud University. In 2010, he promoted to associate professor at the same department. Currently, his is an associate professor at the department of Mathematics and Computer Science, Faculty of Sciences; Beirut Arab University. He has published numerous articles and proceedings in the areas of image processing, remote sensing, and computer vision. He received a B.Sc. in computer science from the Lebanese University; Beirut, Lebanon in 1990, M.Sc. degree in computer science from the University of Sherbrooke, Sherbrooke, Canada in 1996, and Ph.D. degree in computer science from the University of Sherbrooke, Sherbrooke, Canada in 2001. His research interests include image processing, pattern recognition, remote sensing, and computer vision. 